\title{
INNER FUNCTIONS IN POLYDISCS ${ }^{1}$
}

\author{
BY WALTER RUDIN
}

Communicated by A. Zygmund, November 21, 1966

For $N=2,3,4, \cdots$ the polydisc $U^{N}$ consists of all $z=\left(z_{1}, \cdots, z_{N}\right)$ in $C^{N}$ (the space of $N$ complex variables) such that $\left|z_{j}\right|<1$ for $j=1$, $\cdots, N$. The class of all bounded holomorphic functions in $U^{N}$ is denoted by $H^{\infty}\left(U^{N}\right)$. If $f \in H^{\infty}\left(U^{N}\right)$ it is well known that the radial limits

$$
f^{*}(z)=\lim _{r \rightarrow 1} f(r z)
$$

exist for almost all $z$ in the distinguished boundary $T^{N}$ of $U^{N}$. Here $r z=\left(r z_{1}, \cdots, r z_{N}\right)$.

An inner function in $U^{N}$ is, by definition, a function $g \in H^{\infty}\left(U^{N}\right)$ such that $\left|g^{*}(z)\right|=1$ for almost all $z \in T^{N}$.

The present note contains partial answers to questions such as the following: Is every $f \in H^{\infty}\left(U^{N}\right)$ (other than $\left.f \equiv 0\right)$ a product $f=g h$ where $g$ is inner and both $h$ and $1 / h$ are holomorphic in $U^{N}$ ? (In this case we say that $f$ and $g$ have the same zeros in $U^{N}$.) If not, what are some sufficient conditions on $f$ which guarantee the existence of such a factorization? If $f$ does have the same zeros as some inner function $g$, does it follow that $g$ can be chosen so that $f / g \in H^{\infty}\left(U^{N}\right)$ ?

A special role is played by those inner functions which (for lack of a better name) I propose to call good: An inner function $g$ is good if

$$
\lim _{r \rightarrow 1} \int_{T^{N}} \log |g(r z)| d m(z)=0 .
$$

Here $d m$ denotes the Haar measure of $T^{N}$.

To see some examples, consider these four classes of inner functions in $U^{N}$ :

(A) Those which have continuous extensions to the closure of $U^{N}$.

(B) Rational inner functions.

(C) Finite or infinite (convergent) products of rational inner functions.

(D) Good inner functions.

In one variable, $(A)=(B)$ and $(C)=(D)$, since the good inner func-

\footnotetext{
1 Research sponsored by the Air Force Office of Scientific Research, Office of Aerospace Research, United States Air Force, under AFOSR Grant No. 1160-66, and by the Wisconsin Alumni Research Foundation.
} 
tions in one variable are precisely the Blaschke products. That (A) $\subset(\mathrm{B})$ in the general case is proved in [2]. For $N \geqq 2,(\mathrm{~A}) \neq(\mathrm{B})$, as shown by

$$
g(z, w)=(4 z w-3 z-w) /(4-3 w-z) .
$$

It is easy to prove that (B) $\subset$ (D) and hence that (C) $\subset(D)$; in fact, every convergent product of inner functions is inner, and if each factor is good so is the product. For $N \geqq 2,(\mathrm{C}) \neq(\mathrm{D})$ : Let $\Phi$ be any inner function in one variable, let $\alpha$ be a complex number, $0<|\alpha|<1$, and put

$$
g(z, w)=(z \Phi(w)-\alpha) /(1-\bar{\alpha} z \Phi(w)) .
$$

That $g$ is good follows quite easily via Jensen's formula; one can choose $\Phi$ so that the zero-set of $g$ is not a countable union of algebraic varieties, and then $g \in(\mathrm{C})$.

A continuous function in $U^{N}$ is called $N$-harmonic if it is harmonic in each of the variables $z_{1}, \cdots, z_{N}$. If $\Phi \in L^{1}\left(T^{N}\right)$ and if $\mu$ is a measure on $T^{N}$, the Poisson integrals $P[\Phi]$ and $P[d \mu]$ are $N$-harmonic in $U^{N}$ $[4 ;$ pp. 303,315$]$. If $f \in H^{\infty}\left(U^{N}\right)$ and $f \neq 0$, put $f_{r}(z)=f(r z) \quad(0 \leqq r<1$, $\left.z \in T^{N}\right)$ and define

$$
u[f]=\lim _{r \rightarrow 1} P\left[\log \left|f_{r}\right|\right] .
$$

The limit exists (compare [4; pp. 321-322]) and is the least $N$ harmonic majorant of $\log |f|$.

For inner functions $g, u[g] \leqq 0$, and $g$ is good if and only if $u[g]=0$.

We let RP denote the class of all functions $u$ which are real parts of holomorphic functions $u+i v$ in $U^{N}$. Every $u \in \mathrm{RP}$ is clearly $N$ harmonic.

After these preliminaries we can state some results. The first two are quite easy:

THEOREM 1. If $f \in H^{\infty}\left(U^{N}\right), g$ is a good inner function in $U^{N}$, and $\mathrm{f} / \mathrm{g}$ is holomorphic in $U^{N}$, then $\mathrm{f} / \mathrm{g} \in H^{\infty}\left(U^{N}\right)$.

Theorem 2. Suppose $f \in H^{\infty}\left(U^{N}\right), f \not \equiv 0$.

(a) If $u[f]$ is in RP then there is a unique (up to multiplication by constants) good inner function $g$ which has the same zeros as $f$.

(b) If $u[f]$ is not in $\mathrm{RP}$ then no good inner function has the same zeros as $f$.

Theorem 3. Suppose $\Phi \in L^{1}\left(T^{N}\right), \Phi>0$, and $\Phi$ is lower semicontinuous. Then there is a singular positive measure $\sigma$ on $T^{N}$ such that the Poisson integral $P[\Phi-d \sigma]$ is in RP. 
Proof. $\Phi=\sum \Phi_{k}$, where each $\Phi_{k}$ is a positive trigonometric polynomial on $T^{N}$. Denote the Fourier coefficients of $\Phi_{k}$ by $\hat{\Phi}_{k}\left(n_{1}, \cdots, n_{N}\right)$. Fix $k$. If $p_{k}$ is a positive integer, there is a positive singular measure $\sigma_{k}$ on $T^{N}$ such that

$$
\hat{\sigma}_{k}\left(n_{1}, \cdots, n_{N}\right)=\sum_{j=-\infty}^{\infty} \hat{\Phi}_{k}\left(n_{1}+j p_{k}, \cdots, n_{N}+j p_{k}\right) .
$$

If $p_{k}$ is large enough, $P\left[\Phi_{k}-d \sigma_{k}\right]$ is in RP. Put $\sigma=\sum \sigma_{k}$.

THEOREM 4. If $\psi$ is a bounded positive lower semicontinuous function on $T^{N}$ then there exists $f \in H^{\infty}\left(U^{N}\right)$ with $\left|f^{*}\right|=\psi$ a.e.

Proof. The hypothesis implies that $\psi$ has a positive lower bound. Assume $\psi>1$, without loss of generality. Apply Theorem 3 to $\Phi=\log \psi$, put $u=P[\Phi-d \sigma]$, and define $f=\exp (u+i v)$. Note that $P[d \sigma]$ has radial limit 0 a.e. since $\sigma$ is singular $[4 ;$ p. 313].

The assumed lower semicontinuity of $\psi$ is of course not a necessary condition for the existence of an $f \in H^{\infty}\left(U^{N}\right)$ with $\left|f^{*}\right|=\psi$ a.e. Nevertheless it is not an entirely unnatural hypothesis since a certain amount of lower semicontinuity is forced on $\left|f^{*}\right|:$ If $f \in H^{\infty}\left(U^{N}\right)$, $z \in T^{N}$, and $f_{z}(\lambda)=f(\lambda z)$, then $f_{z} \in H^{\infty}(U)$, so that

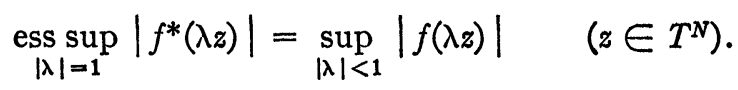

The right side of (7) is clearly a lower semicontinuous function on $T^{N}$, hence so is the left.

TheOREM 5. Suppose $f \in H^{\infty}\left(U^{N}\right), f \not \equiv, \psi$ is an upper semicontinuous function on $T^{N}$, and $\left|f^{*}\right|=\psi$ a.e. Then there is an inner function $g$ with the same zeros as $f$.

Proof. By Theorem 3, applied to $\Phi=-\log \psi$, there is a positive singular measure $\sigma$ on $T^{N}$ such that

$$
u=P[\log \psi+d \sigma]
$$

is in RP. If $h=\exp (u+i v)$, then $\left|h^{*}\right|=\left|f^{*}\right|$ a.e., and

$$
\log |f| \leqq P\left[\log \left|f^{*}\right|\right]=P[\log \psi] \leqq u=\log |h| .
$$

Put $g=f / h$.

The theorem applies, in particular, to any $f \in H^{\infty}\left(U^{N}\right)$ which has a continuous extension to the closure of $U^{N}$. But it may be impossible to choose $g$ so that $f / g$ is bounded in $U^{N}$, even if $f$ is a polynomial! To state this more precisely, let $V^{N}$ be the set of all $\left(z_{1}, \cdots, z_{N}\right)$ with $\left|z_{j}\right|>1$ for $j=1, \cdots N$. 
Theorem 6. Let $f$ be a polynomial in $z_{1}, \cdots, z_{N}, f \neq 0$.

(a) If $f$ has no zero in $V^{N}$ then there is a rational inner function $g$ such that $\mathrm{f} / \mathrm{g}$ is a polynomial with no zero in $U^{N}$.

(b) If $f$ is irreducible and if $f$ has zeros in both $U^{N}$ and $V^{N}$ then $f / g$ is unbounded in $U^{N}$ for every inner function $g$ which has the same zeros as $f$ in $U^{N}$.

Proof. (a) is trivial (see [2; p. 991]). To prove (b), assume $f / g$ is bounded in $U^{N}$, so $|g| \geqq c|f|$ for some $c>0$. Hence, for almost all $z \in T^{N}, g(\lambda z)$ is a finite Blaschke product. This implies (via Theorem 2.1 of [2]) that $g$ is rational. Since $f$ is irreducible it follows that every zero of $f$ (in $C^{N}$ ) is a zero of $g$. But rational inner functions have no zeros in $V^{N}$.

Note that (b) may hold even if $f$ has no zero on $T^{N}: f(z, w)=z+2 w$.

The next result should be compared with Theorem A of [3].

THEOREM 7. There exists $f \in H^{\infty}\left(U^{2}\right), f \neq 0$, with the following property: For no holomorphic function $h$ in $U^{2}$ is fh an inner function.

Proof. Let $A$ be an open set on the unit circle whose complement is totally disconnected and has positive measure, let $E$ be the set of all $(z, w) \in T^{2}$ such that $z / w \in A$. By Theorem 4 there exists $F \in H^{\infty}\left(U^{2}\right)$ with $\left|F^{*}\right|=1$ a.e. on $E,\left|F^{*}\right|=\frac{1}{2}$ a.e. off $E$. A generalization of a theorem of Frostman [1; pp. 111-113] shows that there exist arbitrarily small $\alpha$ such that

$$
u[F-\alpha]=P\left[\log \left|F^{*}-\alpha\right|\right] .
$$

Put $f=F-\alpha$, and suppose (to get a contradiction) that $f h$ is inner for some holomorphic $h$. By $(10), u[f]$ is bounded below, so $u[h]$ is bounded above, hence $h \in H^{\infty}\left(U^{N}\right)$. But $\left|h^{*}\right|$ is close to 1 a.e. on $E$ and $\left|h^{*}\right|$ is close to 2 a.e. on the rest of $T^{N}$. This violates the lower semicontinuity property discussed after Theorem 4.

A more detailed discussion of these results, including complete proofs and extensions to other $H^{p}$-spaces, will be published elsewhere.

\section{REFERENCES}

1. O. Frostman, Potentiel d'équilibre et capacité des ensembles, Lunds Univ. Mat. Sem. Vol. 3 (1935).

2. W. Rudin and E. L. Stout, Boundary properties of functions of several complex variables, J. Math. Mech. 14 (1965), 991-1006.

3. W. Rudin, Zeros and factorizations of holomorphic functions, Bull. Amer. Math. Soc. 72 (1966), pp. 1064-1067. 1959.

4. A. Zygmund, Trigonometric series, Vol. II, Cambridge Univ. Press, New York,

UNIVERSITY OF WISCONSIN AND

University of California, San Diego 